\title{
Distributed Radar Information Fusion for Gait Recognition and Fall Detection
}

\author{
Haobo Li, Julien Le Kernec \\ James Watt School of Engineering \\ University of Glasgow \\ Glasgow, United Kingdom \\ h.li.4@research.gla.ac.uk; \\ Julien.Lekernec@glasgow.ac.uk
}

\author{
Ajay Mehul, \\ Sevgi Zubeyde Gurbuz \\ Department of Electrical and Computer \\ Engineering \\ University of Alabama \\ Tuscaloosa, USA \\ ajaymehu12013@gmail.com; \\ sevgigurbuz@gmail.com
}

\author{
Francesco Fioranelli \\ Microwave Sensing Signals \& Systems \\ (MS3), Department of Microelectronics \\ Delft University of Technology \\ Delft, The Netherlands \\ F.Fioranelli@tudelft.nl
}

\begin{abstract}
This paper discusses a fusion framework with data from multiple, distributed radar sensors based on conventional classifiers, and transfer learning with pre-trained deep networks. The application considered is the classification of gait styles and the detection of critical accidents such as falls. The data were collected from a network comprised of one Ancortek FMCW radar and three UWB Xethru radars. The radar systems within the network were placed in three different locations, notably, in front of participants, on the ceiling, and on the right-hand side of the monitored area. The proposed information fusion framework compares feature level fusion, soft fusion with the classifier confidence level, and hard fusion with Naïve Bayes combiner (NBC). Regarding the classifier, linear SVM, Random-Forest Bagging Trees, and five pretrained neural networks are introduced to the fusion algorithm, where the VGG-16 network yields the best performance (about $84 \%$ ) with the help of NBC. Compared to the best cases with conventional classifiers, it is reported that $20 \%$ and $16 \%$ subsequent improvement are achieved for individual usage of single radar and fusion.
\end{abstract}

Keywords - radar network, information fusion, multiple radar sensing, machine learning, transfer learning

\section{INTRODUCTION}

The growing aging population [1] in western countries and Asia creates significant challenges in providing comprehensive medical care to elderly people with underlying health conditions and timely support after a critical accident such as fall and stroke. Falls usually cause physical injuries [2], [3] including head trauma, face, and hip fracture. These lead to further psychological problems [2], [4] like loss of interest in exercise and fear of being alone. In the UK, the National Health Service (NHS) spent more than 4 million pounds per day to hospitalize the elderly over $65 \mathrm{~s}$ that experienced an accidental fall [5], and this budget has to increase year-by-year because fall may trigger other chronic issues. Researchers found that the life expectancy [6] of the elderly is highly related to the waiting time to receive assistance after the accidents. Thus, a fast-responding and reliable fall detection system [7] can notify the emergency department in the hospital or personal caregivers to provide prompt help. Furthermore, increased fall risk and health anomalies in older people have been correlated with changes in their gait patterns [6], [8]-[10], and related metrics such as asymmetries, slower and less continuous gait, and shorter stride.

The authors acknowledge the support of the UK EPSRC (grant INSHEP EP/R041679/1), University of Glasgow mobility scholarship for PGR students, and GBCET Great Britain China Educational Trust) support for part of the last year of Ph.D. studies of Haobo Li.
Radar is irreplaceable as a contactless sensing technology in many outdoor applications in defense and security. Recently radar has also gained much interest in the context of indoor ambient assisted living [11]. Compared to wearable devices [12], [13] and image/video sensors [14], radar can avoid issues of users' compliance and acceptance due to privacy (no plain images of people or private environments are collected) and/or comfort (no devices to wear, carry, recharge). Radar can also work in through-wall conditions in indoor environments [15] and can provide an estimate of the physical distance and velocity (measured by the Doppler Effect) over time for the monitored subjects [16]

Given the many different types and styles of gaits, people can show while they move, developing radar-based capable classification algorithms is a fundamental challenge. Convolutional Neural Network (CNN) [17] has shown higher potential than conventional classifiers in terms of their classification accuracy. However, those improvements come at a price. They require large amounts of data and computational load as more layers are added which in turn increase the number of hyper-parameters to tune [18].Transfer learning frameworks have been applied to address this issue. Pre-trained networks such as AlexNet (2012)[19], GoogLeNet (2014)[19], VGG-16 (2016) [20] and ResNet family (2018) have been utilized in classifying radar spectrograms and cepstrum maps, exploiting the strength that they gained from prior pre-training with hundred thousands of optical images. This transfer learning method suits for a small experimental radar dataset that would not be enough for feeding and training from scratch a traditional CNN.

In this paper, we investigate the transfer learning framework combined with information fusion from a network consisting of four independent but synchronized radar sensors. The sensors are not clocked by the same reference, but they operate simultaneously and collect data at the same time allowing comparisons as a function of spatial position (three identical UWB X-band radar located at three different positions with respect to the subject) as well as of radar frequency ( 2 co-located radar sensors operate at different frequencies, namely X-band and K-band). In this initial study, we focus on comparing different pre-trained deep network architectures with information fusion schemes, including feature fusion [11], soft decision fusion, and hard decision fusion [21]. The dataset used for the analysis contains 12 different types of gait performed by 14 volunteers, with more details given in the following sections. The initial performance results show that transfer learning can outperform conventional classifiers using manual features and that fusing 
information from the distributed radar sensors in the network is also beneficial.

The remainder of this paper is organized as follows: Section II introduces the radar network setup and describe the gait data collection. Section III discusses the data processing and classification using a conventional classifier and pretrained nets. Section IV presents the results of different information fusion approaches. Finally, section V concludes the paper and outlines possible directions for future work.

\section{EXPERIMENTAL SETUP}

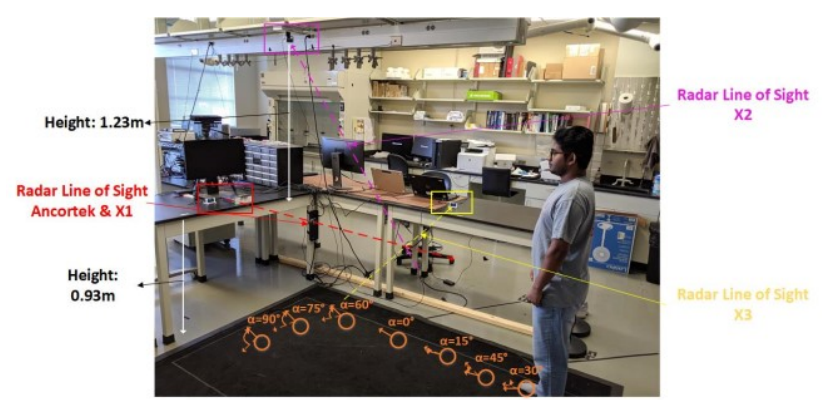

Fig. 1. Experimental setup and walking trajectory. Red line: Ancortek and Xethru in front of the participants; purple line: Xethru on the ceiling; yellow line: Xethru on the right hand side; orange dots on the ground: elliptical trajectory performed by the subjects.

TABLE I. LIST OF THE DIFFERENT GAITS TO BE CLASSIFIED

\begin{tabular}{|l|l|}
\hline G1 & Walking normally \\
\hline G2 & Walking quickly \\
\hline G3 & Walking slowly \\
\hline G4 & Dragging one foot \\
\hline G5 & Limping with an orthopedic cast \\
\hline G6 & Small steps \\
\hline G7 & Walking with a cane \\
\hline G8 & Walking with a walker \\
\hline G9 & Military walking \\
\hline G10 & Bunny jump \\
\hline G11 & Walking and direct fall \\
\hline G12 & Walking and controlled fall \\
\hline
\end{tabular}

The dataset was collected in the Computational Intelligence for Radar (CI4R) Lab at the University of Alabama; it contains 11 male and three female participants aged from 19 to 44 . Table I lists twelve different gaits, including walking with different speed, dragging one foot while walking, moving with small steps, walking with aids, jumping back and forth, as well as some joint gaits (gaits followed by a fall event). In the experiment, the participants are asked to perform $20 \mathrm{~s}$ elliptical loops in different gait styles (with the lab setup and the trajectory shown in Fig. 1), whereas in 'G11' and 'G12', two kinds of falling are following a short period of walking (12s approximately) to attempt to simulate the sudden loss of consciousness and progressive exhaustion and fall of elderly people, respectively.

One Ancortek FMCW radar operating at $25 \mathrm{GHz}$ and three Novelda Xethru UWB Doppler radars at $7.5 \mathrm{GHz}$ are utilized to measure the gait patterns, simultaneously with three different spatial perspectives in Fig. 1. The Ancortek radar and one of the Xethru radars (X1) are set on the table in front of the participants (red box in Fig. 1); the second Xethru (X2) is fixed on the ceiling with an elevation angle of about $45^{\circ}$ to the center of the experimental zone (purple box in Fig. 1); the last
Xethru (X3) is placed at the right-hand side of the participant (yellow box in Fig. 1). The FMCW radar transmits a chirp signal with $2 \mathrm{GHz}$ bandwidth and $1 \mathrm{kHz}$ PRF (Pulse Repetition Frequency), whereas the impulse Doppler radar has $1.5 \mathrm{GHz}$ bandwidth with $500 \mathrm{kHz}$ PRF.

The radar network is constructed by connecting all the individual radar to a laptop via USB cables. It is synchronized by adding a delay function as the two types of radar have different waking up time to compensate. This allows recording simultaneously data from all four radars, although the radars are not coherently synchronized by the same clock. Besides that, data from the pressure mattress on the floor in Fig. 1 is also collected and can be used as ground truth for the location of the subject and to examine the sequence of the steps in different gaits.

The dataset is saved in a format of $m^{*} n^{*} q$, where $m$ is the number of participants, $n$ is the gait class and $q$ is the number of the $20 \mathrm{~s}$ long repetitions of each gait; in our case, $q$ equals to 3 , hence, so the total number of observation is 504 $(14 \cdot 12 \cdot 3)$.

\section{DATA PROCESSING AND ANALYSIS}

\section{A. Conventional Classifier}

The radar data can be mapped into three different domains, notably, Range-Time, Range-Doppler, and Doppler-Time, also known as a radar cube when combined [16]. The RangeTime matrix is obtained by applying a $1^{\text {st }}$ Fast Fourier Transform (FFT) on the raw amplitude and phase of the radar data, whereas the Range-Doppler maps are generated by using a $2^{\text {nd }}$ FFT along the time axis of the Range-Time matrix for each range bin. The Doppler-Time domain also referred as a spectrogram, is generated by adding the range bins together for each time bin and then using a Short-Time Fourier Transform (STFT) successivelyto visualize the microDoppler signature, which is significant in characterizing periodic motions such as swinging of human legs and arms. Fig. 2 shows the radar spectrograms of different gaits, where the positive Doppler shift represents the stride towards the radar and vice versa. In this paper, we focus on the spectrogram analysis; the window function used in the STFT is a hamming window with $0.2 \mathrm{~s}$ length and $95 \%$ overlapping.

Some statistical features are extracted to replace the whole spectrogram as the input of the classifier. The radar features [22], [23] used in this paper are listed in Table II, they can be divided into physical features and transform-based features, where the physical features include upper envelope, lower envelope (shown in Fig. 2 with red and white lines), centroid and bandwidth of the Doppler spectrogram. Differently from those, transform-based features perform a mathematic transformation (e.g., SVD (Singular Value Decomposition), DCT (Discrete Cosine Transform) and LPC (Linear Predictive Coding) on the spectrogram data to find more information on a specific dimension. Similar features are also generated from Cadence Velocity Diagram (CVD) and radar cepstrum.

Two robust conventional classifiers, notably, linear SVM and Random-Forest (RF) bagging with 200 trees are selected to train the prediction model and evaluate the classification performance. In this paper, a 'Leaving one participant out' (L1O) cross-validation method is used to partition the dataset into training and test part, where data from one participant is chosen to evaluate the classification performance, and the rest of the data is used to train the classifier. Every subject in the 
dataset is, in turn, the 'test participant', and the results are averaged from the 14 iterations. Compared to the conventional ' $\mathrm{k}$-fold' or simpler 'holdout,' L1O successfully simulates the more realistic scenario that the classifier cannot access all subjects' data prior to the actual usage, i.e., the classifier needs to deal well with unknown new subjects.
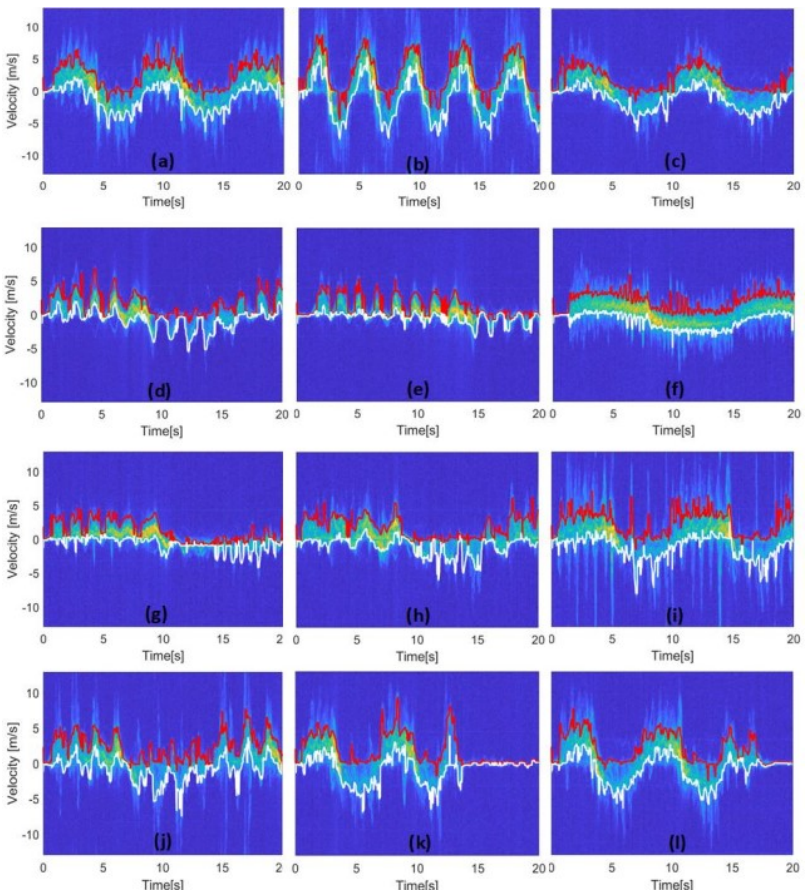

Fig. 2. Ancortek radar spectrograms. The spectrogram from (a) to (i) correspond to the gait from ' $\mathrm{G} 1$ ' to ' $\mathrm{G} 12$ ' in Table I. Red line: upper envelope, white line: lower envelope.

\section{TABLE II.}

LIST OF THE RADAR MANUAL FEATURES

\begin{tabular}{|c|c|}
\hline Physical features & $\begin{array}{l}\text { No. of } \\
\text { features }\end{array}$ \\
\hline $\begin{array}{l}\text { Mean, standard deviation, skewness, and kurtosis of the } \\
\text { centroid of the Doppler spectrogram }\end{array}$ & 4 \\
\hline $\begin{array}{l}\text { Mean, standard deviation, skewness, and kurtosis of the } \\
\text { bandwidth of the Doppler spectrogram }\end{array}$ & 4 \\
\hline $\begin{array}{l}\text { Two-dimensional mean, standard deviation, skewness } \\
\text { and kurtosis of the whole segment of the spectrogram }\end{array}$ & 4 \\
\hline Mean, maximum and minimum of the upper envelope & 3 \\
\hline Mean, maximum and minimum of the lower envelope & 3 \\
\hline $\begin{array}{l}\text { Difference between the mean of the upper and lower } \\
\text { envelope }\end{array}$ & 1 \\
\hline Transform-based features & $\begin{array}{l}\text { No. of } \\
\text { features }\end{array}$ \\
\hline $\begin{array}{l}\text { Mean and standard deviation of the first left and right } \\
\text { eigenvector of the SVD decomposition of the } \\
\text { spectrogram }\end{array}$ & 4 \\
\hline Sum of pixels of the entire left and right matrices & 2 \\
\hline Mean of the diagonal of the left and right matrices & 2 \\
\hline Discrete DCT of the spectrogram & 10 \\
\hline First 10 coefficients of the LPC of the spectrogram & 10 \\
\hline Step repetition frequency & 1 \\
\hline Step repetition frequency band peak & 2 \\
\hline Intensity of the main peak in CVD & 1 \\
\hline Maximum of the main peak & 1 \\
\hline Energy of the main peak & 1 \\
\hline Most significant Doppler frequency in CVD & 1 \\
\hline Maximum, minimum and mean of the cepstrum & 3 \\
\hline Total number of features & 57 \\
\hline
\end{tabular}

The L1O classification results of using radar individually are summarized in Table III, where the Xethru radar in front of the participant outperforms other radars with SVM, and the
Xethru radar on the ceiling yields the best performance with RF Bagging Trees. There is not much difference in Ancortek and $\mathrm{X} 1$ using these two classifiers, whereas $\mathrm{X} 2$ and $\mathrm{X} 3$ share a $5 \%$ improvement. Fig. 3 shows in a confusion matrix the misclassification rates between each class, where the rows are output classes, and the columns represent target classes. The diagonal elements are the gaits that are correctly classified, whereas the non-diagonal elements denote the misclassified gaits. The sum of the elements in each column is equal to $100 \%$. This confusion matrix reports high misclassification in 'G1', 'G3', 'G5', and 'G7', especially between 'G1' and 'G3'. The walking speed of for different people varies, and may cause the algorithm to classify 'slow walking' for some subjects as 'normal walking' for others. The same reason would explain the misclassifications between ' $\mathrm{G} 5$ ' and 'G7', as those gaits are carefully chosen to be similar in pairs for creating more classification challenge. For the last two joint gaits which contain a fall event, the correctly classified rate is not too low. However, there are some false alarms with other classes, and this affects a lot in recognizing critical events like falls.

TABLE III. THE L1O CLASSIFICATION RESULTS FOR SINGLE RADAR SENSORS (MAX, Min, MEAN AND STANDARD DEVIATION)

\begin{tabular}{|c|c|c|c|c|}
\hline $\begin{array}{c}\text { Linear } \\
\text { SVM }\end{array}$ & Ancortek & X1 & X2 & X3 \\
\hline Mean & $58.53 \%$ & $\mathbf{5 9 . 1 3 \%}$ & $58.33 \%$ & $49.6 \%$ \\
\hline Max & $80.56 \%$ & $80.56 \%$ & $72.22 \%$ & $66.67 \%$ \\
\hline Min & $36.11 \%$ & $19.44 \%$ & $38.89 \%$ & $25 \%$ \\
\hline STD & 0.1393 & 0.165 & $\mathbf{0 . 1 0 1}$ & 0.1293 \\
\hline $\begin{array}{c}\text { RF } \\
\text { Bagging }\end{array}$ & Ancortek & $\mathbf{X 1}$ & $\mathbf{X 2}$ & $\mathbf{X 3}$ \\
\hline Mean & $59.52 \%$ & $59.72 \%$ & $\mathbf{6 3 . 4 9 \%}$ & $54.17 \%$ \\
\hline Max & $75 \%$ & $77.78 \%$ & $83.33 \%$ & $77.78 \%$ \\
\hline Min & $38.89 \%$ & $36.11 \%$ & $44.44 \%$ & $33.33 \%$ \\
\hline STD & 0.1246 & 0.1241 & 0.1251 & 0.1172 \\
\hline
\end{tabular}

\section{B. Transfer Learning using Pre-trained Networks}

Transfer learning [19], [20] has attracted lots of interest in the field of image classification in applications such as face and gesture recognition. Fig. 4 illustrates the training and testing scheme of the transfer learning with a VGG-16 net taken as an example. It uses the output weights from a deep neural network pre-trained on numerous optical images, which enables the network to capture the common concepts among the edges, curves, and other properties of the figure patterns. This can lead to a potential application that makes this network capable of adapting to a new dataset by retraining with a small amount of the new labeled data, radar data in this case, and fine-tuning the original weights.

Transfer learning uses a pre-trained network, and this solves two specific issues of the classic Convolution Neural Network (CNN). Notably, it does not require a large dataset to train, and as a result of that, it saves a lot of training time and computational load. In this paper, five pre-trained networks, notably AlexNet, GoogLeNet, VGG-16, ResNet18, and ResNet101, are empirically selected to re-train with radar spectrograms and compare the classification performance.

Table IV lists the classification performance of each radar using a pre-trained net in terms of mean, maximum, minimum, and standard deviation across the leave one participant out (L1O) tests for the 14 subjects. From the perspective of average performance, VGG-16 outperforms the other pre- 
trained networks, where the Xethru radar on the ceiling yields the best results among all the available radars. It is reported that Ancortek radar using ResNet101 provides better average performance than VGG-16, which seems to show that ResNet101 is more powerful in characterizing the features from Ancortek images.

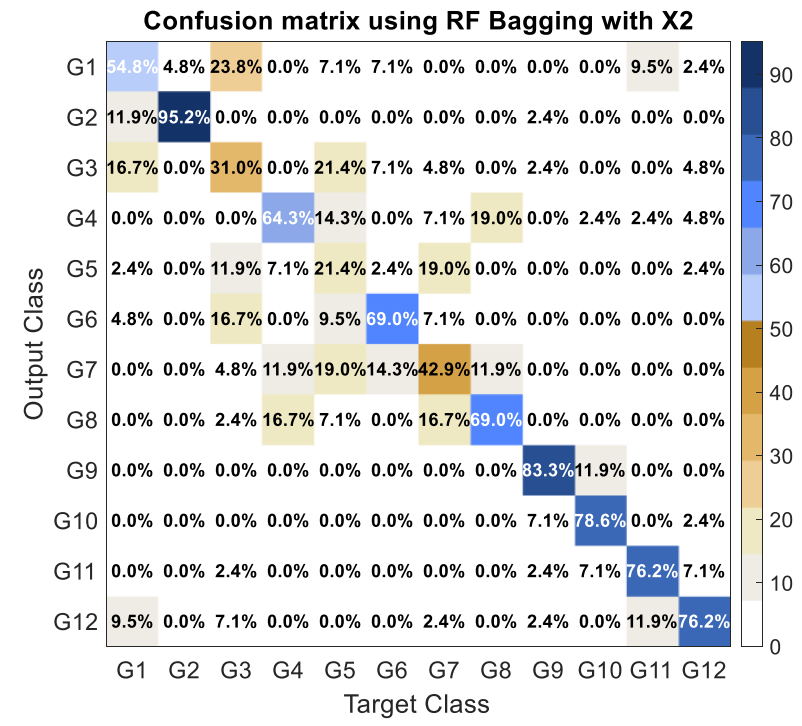

TABLE IV. THE L1O CLASSIFICATION RESULTS USING PRETRAINED NETWORKS ON DATA FROM SINGLE RADAR SENSORS

\begin{tabular}{|l|l|l|l|l|}
\hline Alexnet & Ancortek & $\mathbf{X 1}$ & $\mathbf{X 2}$ & $\mathbf{X 3}$ \\
\hline Mean & $67.86 \%$ & $71.83 \%$ & $71.83 \%$ & $64.88 \%$ \\
\hline Max & $83.33 \%$ & $86.11 \%$ & $91.67 \%$ & $86.11 \%$ \\
\hline Min & $50 \%$ & $47.22 \%$ & $44.44 \%$ & $38.89 \%$ \\
\hline STD & 0.1179 & 0.1308 & 0.1517 & 0.1483 \\
\hline VGG-16 & Ancortek & $\mathbf{X 1}$ & $\mathbf{X 2}$ & $\mathbf{X 3}$ \\
\hline Mean & $73.41 \%$ & $75.2 \%$ & $79.96 \%$ & $71.23 \%$ \\
\hline Max & $91.67 \%$ & $91.67 \%$ & $94.44 \%$ & $83.33 \%$ \\
\hline Min & $47.22 \%$ & $47.22 \%$ & $63.89 \%$ & $50 \%$ \\
\hline STD & 0.1202 & 0.1372 & 0.1069 & 0.1236 \\
\hline GoogLeNet & Ancortek & $\mathbf{X 1}$ & $\mathbf{X 2}$ & $\mathbf{X 3}$ \\
\hline Mean & $66.47 \%$ & $60.52 \%$ & $62.9 \%$ & $55.95 \%$ \\
\hline Max & $80.56 \%$ & $83.33 \%$ & $80.56 \%$ & $75 \%$ \\
\hline Min & $47.22 \%$ & $44.44 \%$ & $47.22 \%$ & $38.89 \%$ \\
\hline STD & 0.1114 & 0.1199 & 0.1048 & 0.1050 \\
\hline ResNet18 & $\mathbf{A n c o r t e k}$ & $\mathbf{X 1}$ & $\mathbf{X 2}$ & $\mathbf{X 3}$ \\
\hline Mean & $71.83 \%$ & $63.89 \%$ & $66.27 \%$ & $60.52 \%$ \\
\hline Max & $86.11 \%$ & $80.56 \%$ & $80.56 \%$ & $72.22 \%$ \\
\hline Min & $58.33 \%$ & $52.78 \%$ & $55.56 \%$ & $47.22 \%$ \\
\hline STD & 0.0936 & 0.0844 & 0.0864 & 0.0845 \\
\hline ResNet101 & Ancortek & $\mathbf{X 1}$ & $\mathbf{X 2}$ & $\mathbf{X 3}$ \\
\hline Mean & $76.19 \%$ & $68.85 \%$ & $66.87 \%$ & $60.32 \%$ \\
\hline Max & $88.89 \%$ & $83.33 \%$ & $77.78 \%$ & $77.78 \%$ \\
\hline Min & $55.56 \%$ & $52.78 \%$ & $52.78 \%$ & $33.33 \%$ \\
\hline STD & 0.1009 & 0.0831 & 0.0640 & 0.1244 \\
\hline & & & &
\end{tabular}

Fig. 3. Confusion matrix of Xethru P2 using RF Bagging Trees

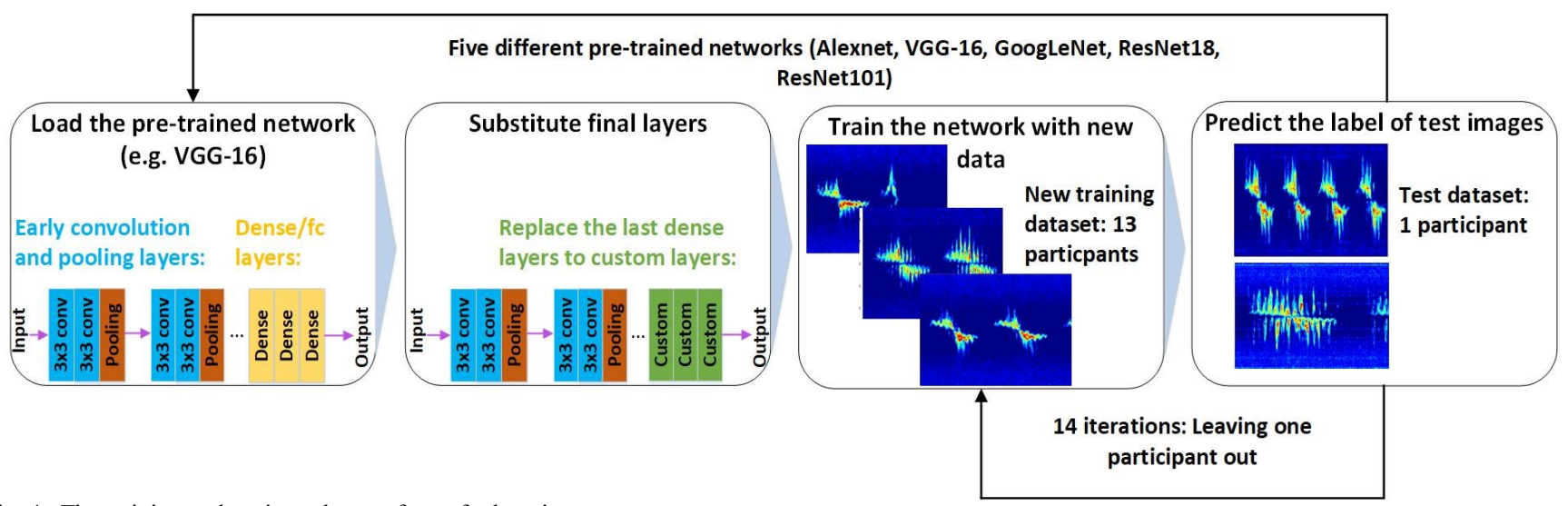

Fig. 4. The training and testing scheme of transfer learning.

\section{INFORMATION FUSION}

In the circumstance that the participant is moving in a large angle with respect to the radar line-of-sight, the receiving signal strength is not optimal due to the well-known aspect angle problem. This may lead to possible misclassification and false alarms. However, the low classification performance of one radar at a certain time could be mitigated by using the data of other radars working at different frequency bands and placed at different locations in a radar network.

$$
\begin{gathered}
\left|F_{A n}[:] F_{X_{1}}[:] F_{X_{2}}[:] F_{X_{3}}[:]\right|=F_{\text {fusion }}[:] \\
W_{a} \cdot S_{A n}(n, c)+W_{b} \cdot S_{X_{1}}(n, c)+W_{c} \cdot S_{X_{2}}(n, c) \\
+W_{d} \cdot S_{X_{3}}(n, c)=S_{\text {fusion }}(n, c) \\
P\left(C_{k} \mid d\right)=P\left(C_{k}\right) \cdot \prod_{m=1}^{N} p_{m, R_{m}, k}
\end{gathered}
$$

The fusion of radar data, can take place at feature and decision level separately. Feature fusion physically cascades the feature subset from each radar to a feature pool, as in (1). Decision fusion is divided into soft fusion and hard fusion. Soft fusion uses the confidence level of the separate classifiers to generate the new prediction label. If a weighted index is introduced to each radar, then it becomes weighted soft fusion. In (2), $S(n, c)$ refers to the confidence level for observation $n$, and class $c, W_{a}$ to $W_{d}$ denote the weight coefficients for each radar, respectively. Generally, the radar with high individual classification performance is associated with higher weight. Hard fusion relies on the posterior probability of the class of interest in the confusion matrix to make a new decision. Typical hard fusion methods include majority voting, weighted majority voting, recall combiner, and Naive-Bayes (NB) combiner [24]. Eq. (3) shows the mathematical representation of an NB combiner, where $P\left(C_{k}\right)$ is the classifier support rate, $R_{m}$ denotes to the prediction label of classifier $m, k$ is the class of interest. The output is the product of the support rate and the element of the radar confusion matrix (classifier $m$, row $R_{m}$ and column $k$ ). From the results in our previous work[21], NB combiner is chosen as the main 
information fusion approach. Feature level fusion and equal weighted soft fusion are also considered as alternative for comparison with conventional classifier and transfer learning respectively.

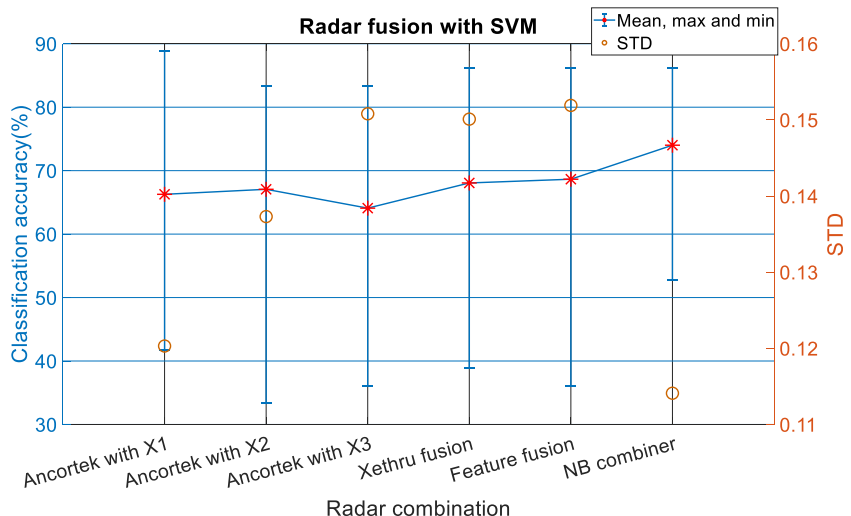

Fig. 5. The statistical parameters of radar fusion with classifiers using linear SVM.

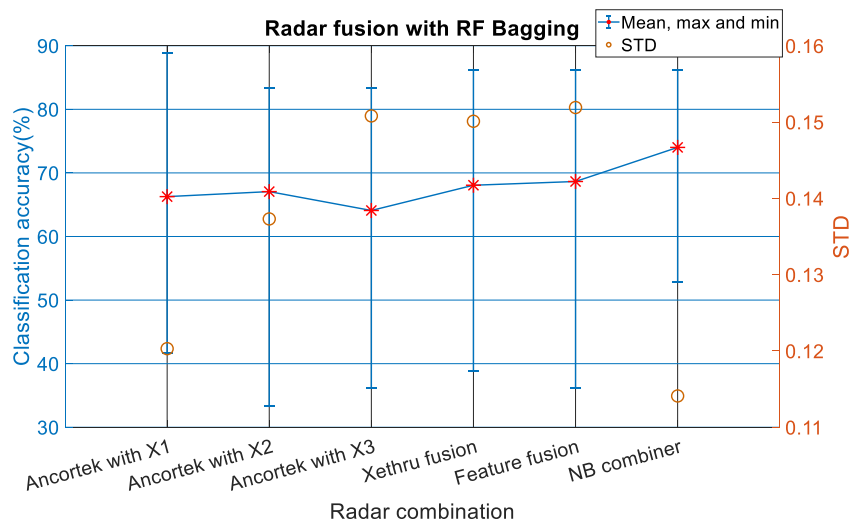

Fig. 6. The statistical parameters of radar fusion with classifiers using RF Bagging Trees.

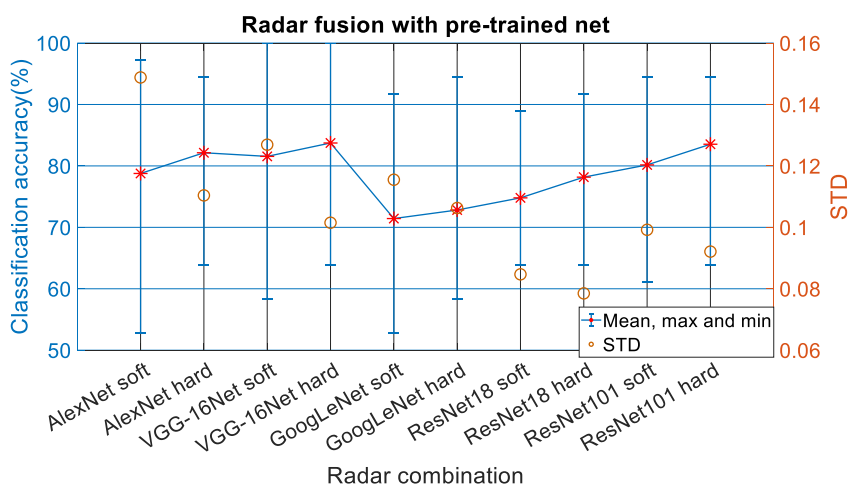

Fig. 7. The statistical parameters of soft and hard fusion with all the radars in the network using transfer learning.

Fig. 5-6 illustrate the statistical parameters of the radar fusion with SVM and RF Bagging Trees. Different combinations of different radars are considered. Note that in the figures, "feature fusion" and "NB combiner" cases included data from all the radar sensors in the network. For the SVM, fusion with all radars with NB combiner yields the best classification performance, approximately $4 \%$ and $19 \%$ higher than the best and worst case in the single radar scenario. Additionally, significant improvement (about $6 \%$ and $7 \%$ ) is reported that combining Ancortek with X1 and X2 at the feature level.

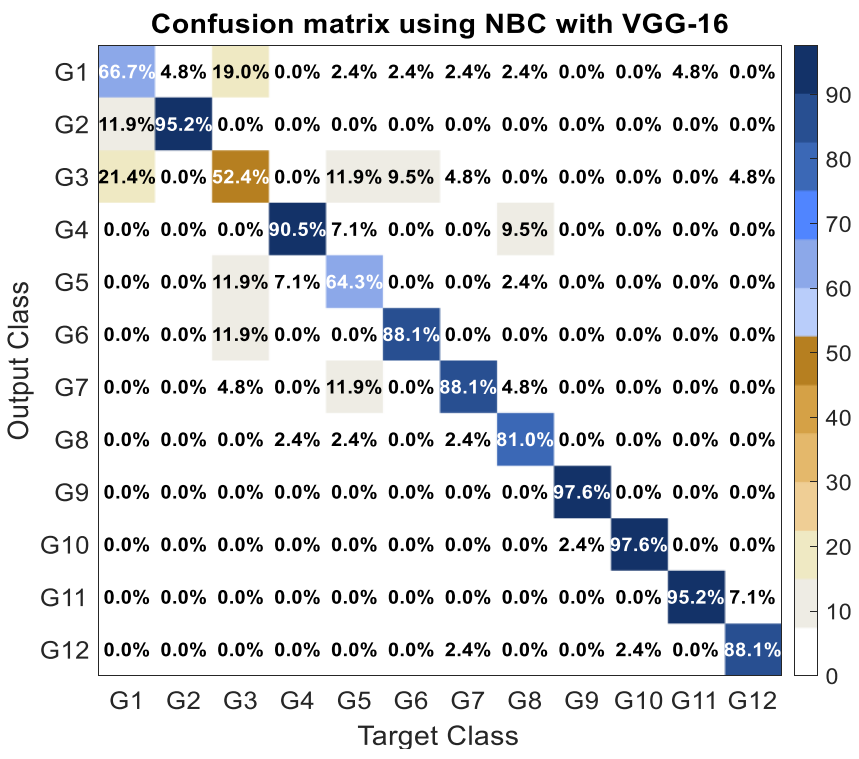

Fig. 8. Confusion matrix of Naïve Bayes combiner hard fusion with VGG16 network.

Fig. 7 shows the same parameters of fusion using a pretrained network, VGG-16 surpasses other networks in both hard and soft fusion, whereas ResNet101 is only $0.5 \%$ lower in hard fusion. The main benefit of transfer learning is increasing the minimum accuracy to $64 \%$, which is $12 \%$ higher than the same approach with RF Bagging Trees. Meanwhile, the standard deviations of transfer learning are lower than conventional classifiers, in other words, the variation of performance by the participants is lower, and this leads to a more stable system.

Fig. 8 discusses the misclassification between classes using NB combiner hard fusion with a VGG-16 pre-trained network. Compared to Fig. 3, the classification accuracy rises to $83.73 \%$, and most of the misclassified events are corrected, whereas the wrong classified gaits between 'G1' and 'G3' are still existing but less than before due to the similarity. The classification rates of ' $\mathrm{G} 5$ ' and ' $\mathrm{G} 7$ ' are improved significantly by $43 \%$ and $46 \%$, respectively. Additionally, the false alarms of falling in 'G11' and 'G12' are much lower than using X2 individually.

\section{CONCLUSION}

This paper presents an information fusion framework applied on radar data from a network of four coordinated sensors, namely three UWB Xethru radar and one FMCW Ancortek radar. Conventional classifiers and transfer learning approaches with five different pre-trained deep networks are compared, with the aim of recognizing different gait styles and identifying fall accidents.

The data from four different radar systems are combined at feature and decision level to provide subsequent improvement of $4-20 \%$ than the best case of using radar individually. It is reported that Naïve Bayes combiner (NBC) based on the posterior probability of the class of interest outperforms other fusion techniques. In terms of the classifier, VGG-16 yields the best classification performance among SVM, RF Bagging Trees, and other pre-trained networks. Data fusion using NBC with VGG-16 indicates approximately $84 \%$ average classification accuracy after considering all the participants as test subjects. 
Future work will evaluate the information fusion method on a wider platform. This includes more participants, more aspect angles with the radar, and even multimodal approaches as seen in [25]. Regarding the neural network, a sequential classification task, as seen in [21] with continuous gaits will be considered as well as the meta-learning of the hyperparameters and structure of the pre-trained network.

\section{ACKNOWLEDGMENT}

The authors are grateful to the colleagues in the CI4R lab for their participation and help in the data collection.

\section{REFERENCES}

[1] W. H. O. Ageing and L. C. Unit, "WHO global report on falls prevention in older age," World Heal. Organ., 2008.

[2] K. Chaccour, R. Darazi, A. H. El Hassani, and E. Andrès, "From fall detection to fall prevention: A generic classification of fall-related systems," IEEE Sens. J., vol. 17, no. 3, pp. 812-822, 2017.

[3] C. S. Florence, G. Bergen, A. Atherly, E. Burns, J. Stevens, and C. Drake, "Medical costs of fatal and nonfatal falls in older adults," J. Am. Geriatr. Soc., 2018.

[4] M. Mubashir, L. Shao, and L. Seed, "A survey on fall detection: Principles and approaches," Neurocomputing, vol. 100, pp. 144-152, 2013.

[5] NIHR Dissemination Centre, "HELP AT HOME Use of assistive technology for older people," Natl. Inst. Heal. Res., pp. 3-6, 2018.

[6] F. Wang, M. Skubic, M. Rantz, and P. E. Cuddihy, "Quantitative Gait Measurement With Pulse-Doppler Radar for Passive In-Home Gait Assessment," IEEE Trans. Biomed. Eng., vol. 61, no. 9, pp. 2434 2443, 2014.

[7] H. Wang, D. Zhang, Y. Wang, J. Ma, Y. Wang, and S. Li, "RT-Fall: A real-time and contactless fall detection system with commodity WiFi devices," IEEE Trans. Mob. Comput., vol. 16, no. 2, pp. 511-526, 2017.

[8] X. Bai, Y. Hui, L. Wang, and F. Zhou, "Radar-Based Human Gait Recognition Using Dual-Channel Deep Convolutional Neural Network," IEEE Trans. Geosci. Remote Sens., vol. 57, no. 12, pp. 9767-9778, 2019.

[9] A. Seifert, M. G. Amin, and A. M. Zoubir, "Toward Unobtrusive InHome Gait Analysis Based on Radar Micro-Doppler Signatures," IEEE Trans. Biomed. Eng., vol. 66, no. 9, pp. 2629-2640, 2019.

[10] A. Seifert, A. M. Zoubir, and M. G. Amin, "Detection of Gait Asymmetry Using Indoor Doppler Radar," in 2019 IEEE Radar Conference (RadarConf), 2019, pp. 1-6.

[11] H. Li et al., "Multisensory Data Fusion for Human Activities Classification and Fall Detection," in 2017 IEEE Sensors Conference, 2017, pp. 1-3.

[12] H. Li, A. Shrestha, H. Heidari, J. L. Kernec, and F. Fioranelli, "Magnetic and Radar Sensing for Multimodal Remote Health Monitoring,” IEEE Sens. J., vol. 19, no. 20, pp. 8979-8989, 2018.

[13] T. R. Bennett, J. Wu, N. Kehtarnavaz, and R. Jafari, "Inertial measurement unit-based wearable computers for assisted living applications: A signal processing perspective," IEEE Signal Process. Mag., vol. 33, no. 2, pp. 28-35, 2016.

[14] D. Wu et al., "Deep Dynamic Neural Networks for Multimodal Gesture Segmentation and Recognition," IEEE Trans. Pattern Anal. Mach. Intell., vol. 38, no. 8, pp. 1583-1597, 2016.

[15] P.-H. Chen, M. C. Shastry, C.-P. Lai, and R. M. Narayanan, "A portable real-time digital noise radar system for through-the-wall imaging," IEEE Trans. Geosci. Remote Sens., vol. 50, no. 10, pp. 4123-4134, 2012.

[16] B. Erol and M. G. Amin, "Radar Data Cube Processing for Human Activity Recognition Using Multi Subspace Learning," IEEE Trans. Aerosp. Electron. Syst., 2019.

[17] Y. Kim and T. Moon, "Human detection and activity classification based on micro-Doppler signatures using deep convolutional neural networks," IEEE Geosci. Remote Sens. Lett., vol. 13, no. 1, pp. 8-12, 2016.

[18] J. Le Kernec et al., "Radar Signal Processing for Sensing in Assisted Living: The challenges associated with real-time implementation of emerging algorithms," IEEE Signal Process. Mag., vol. 36, no. 4, pp. $29-41,2019$.

[19] A. Shrestha et al., "Cross-Frequency Classification of Indoor Activities with DNN Transfer Learning," in 2019 IEEE Radar Conference (RadarConf), 2019, pp. 1-6.

[20] S. Z. Gurbuz and M. G. Amin, "Radar-Based Human-Motion Recognition With Deep Learning: Promising applications for indoor monitoring," IEEE Signal Process. Mag., vol. 36, no. 4, pp. 16-28, 2019.

[21] H. Li, A. Shrestha, H. Heidari, J. Le Kernec, and F. Fioranelli, "BiLSTM Network for Multimodal Continuous Human Activity Recognition and Fall Detection," IEEE Sens. J., vol. 20, no. 3, pp. 1191-1201, 2020

[22] S. Z. Gürbüz, B. Erol, B. Çağlıyan, and B. Tekeli, "Operational assessment and adaptive selection of micro-Doppler features," IET Radar, Sonar Navig., vol. 9, no. 9, pp. 1196-1204, 2015.

[23] F. Fioranelli, M. Ritchie, S. Z. Gürbüz, and H. Griffiths, "Feature diversity for optimized human micro-Doppler classification using multistatic radar," IEEE Trans. Aerosp. Electron. Syst., vol. 53, no. 2, pp. 640-654, 2017.

[24] L. Kuncheva and J. Rodríguez, "A weighted voting framework for classifiers ensembles," Knowledge and Information Systems, vol. 38. 2014.

[25] E. Cippitelli, F. Fioranelli, E. Gambi, and S. Spinsante, "Radar and RGB-depth sensors for fall detection: a review," IEEE Sens. J., vol. 17, no. 12, pp. 3585-3604, 2017. 